\title{
Development of Post Hybrid Calcium Carbonate for High Loaded Paper
}

\author{
Min Woo Lee, ${ }^{\mathrm{a}}$ Dong Suk Kang, ${ }^{\mathrm{b}}$ and Yung Bum Seo ${ }^{\mathrm{c}, *}$ \\ In papermaking, pre-flocculation of fillers such as ground calcium \\ carbonate (GCC) improves the tensile strength of paper sheets. However, \\ the pre-flocculated fillers mostly suffer from the instability of the floc shape \\ such as the decrease in floc diameter with time elapse after preparation \\ and no improvement of bulk and stiffness. The addition of calcium \\ compounds such as calcium oxide or calcium hydroxide to the pre- \\ flocculated GCC, and injection with carbon dioxide caused pre-flocculated \\ GCC flocs to be covered with newly formed calcium carbonate. This \\ product, called post hybrid calcium carbonate (pHCC), was found to be \\ more stable in size and gave better sheet strength than the pre-flocculated \\ ones. Furthermore, pHCC gave remarkably higher bulk and stiffness than \\ the pre-flocculated flocs did without impairing smoothness that was \\ essential in printing paper. The proper use of pHCC in papermaking could \\ allow the production of high loaded paper with more than $10 \%$ higher filler \\ contents, which could reduce paper production cost and save drying \\ energy. The proportion of the newly formed calcium carbonate in pHCC, \\ turbulence intensity at preparation stage, and the effect of storage time \\ were investigated.
}

Keywords: Highly loaded paper; Post hybrid calcium carbonate (pHCC); Ground calcium carbonate (GCC); Pre-flocculation; Calcium oxide; Calcium hydroxide; Bulk; Stiffness; Production cost;

Energy savings

Contact information: a: Hansol Paper Ltd, Daedeok-gu, Daejeon, Republic of Korea; b: Hanchang Paper Co, Yongdang, Yangsan-si, Republic of Korea; c: Department of Bio-based Materials, Chungnam Nat. Univ, Yousung-Gu, Daejeon, Republic of Korea; *Corresponding author: ybseo@cnu.ac.kr

\section{INTRODUCTION}

Pre-flocculation is a process that aggregates papermaking fillers by means of polyelectrolytes. Flocculation of filler particles with polyelectrolytes decreases the specific surface area of the filler particles, and this reduces the loss of the fiber-fiber bonding area. There have been many studies about the flocculation kinetics of filler aggregation (Burgess et al. 2000; Fuente et al. 2005; Lee and Lee 2006; Modgi et al. 2006; Gaudreault et al. 2009). The effects of the characteristics of polyelectrolytes on floc properties have been studied extensively, and the molecular weight of the polymer strongly affects the quality of the flocs (Burgess et al. 2000; Fuente et al. 2005; Gray and Ritchie 2006; Gaudreault et al. 2009).

The hydrodynamic shear rate is an important factor that affects the flocculated particles. Lee and Lee (2006) showed that an increase in the shear rate results in a decrease in the floc size. As for floc quality, the rupture strength of the flocs varied with the hydrodynamic conditions where the flocs were formed (Yeung et al. 1997). Choi and Seo (2016) showed the pre-flocculated GCC (pGCC) resulted in paper having higher tensile strength than the untreated GCC, but there were no increases of bulk and stiffness for the 
pre-flocculated ones. Furthermore, there was decrease in paper opacity. Many reports show that there were increases of tensile strength by the pre-flocculation process, but no increase in bulk (Seo et al. 2012; Chauhan and Bhardwaj 2014; Jung and Seo 2015; Choi and Seo 2016).

Choi et al. $(2016,2018)$ introduced hybrid calcium carbonate (HCC) technology, where GCC and calcium oxide were mixed together in a fixed ratio and were preflocculated together by using polyelectrolytes, and thereafter carbon dioxide was injected to the pre-flocculated flocs to make the aggregates of the GCC and the newly formed calcium carbonate. They called the aggregates HCC. The property of $\mathrm{HCC}$ was similar to the pre-flocculated GCC (pGCC) in tensile strength development, but it gave much higher bulk and stiffness.

Choi and Seo (2016) introduced another technology that was called post hybrid calcium carbonate (pHCC). In the manufacture of pHCC, GCC particles were preflocculated by using polyelectrolytes in the first step, calcium oxide or the calcium hydroxide was added and mixed with the pre-flocculated GCC in the second step, and finally, carbon dioxide gas was injected to the mixture to form the newly formed calcium carbonate on the surface of the GCC flocs. The pHCC of excellent performance usually had the similar size to that of the pGCC and HCC. HCC and pHCC were more rigid than the pGCC, but less deformable than the pGCC under the pressure. These properties of the $\mathrm{HCC}$ and the $\mathrm{pHCC}$ allowed the paper sheet properties to reach high bulk and smoothness levels at the same time (Choi and Seo 2016).

In this study, the properties of pHCC were compared with HCC and pre-flocculated GCC. The manufacture of pHCC was much faster and more versatile than that of the HCC, and it gave possibility of allowing higher stability in its shape and physical properties with time elapse in comparison to the HCC and the pGCC.

\section{EXPERIMENTAL}

\section{Materials and Methods}

The Omya Korea Co. gratefully donated the GCC with its mean diameter of 2.0 $\mu \mathrm{m}$. Calcium oxide was purchased from Showa Chemicals Co. Ltd., Tokyo, Japan. Calcium hydroxide was donated by Baekkwang Mineral Products Co., Ltd. (Danyang, Republic of Korea). As a retention aid for paper sheet preparation, cationic polyacrylamide (C-PAM. MW 5-7 million g/mol. +5 meq/g) from Solenis Chemical (Daejeon, Republic of Korea) was used at $0.1 \%$ dosage based on the dry weight of the GCC added furnish.

To make handsheets, a mixture (20:80) of commercial softwood bleached kraft pulp (SwBKP; a mixture of hemlock, Douglas fir, and cedar) and hardwood bleached kraft pulp (HwBKP; a mixture of aspen and poplar) was used as the wood fiber furnish, both of which came from Canada. These wood pulps were refined together in a laboratory Hollander beater until their freeness reached to $450 \mathrm{~mL}$ CSF. After mixing with the prepared fillers and the refined fiber furnish, handsheets of $60 \mathrm{~g} / \mathrm{m}^{2}$ were made with $30 \%$ and $40 \%$ ash contents. By careful control of the handsheet making process, basis weight of the handsheets came out uniformly $\left(60.0 \pm 1.5 \mathrm{~g} / \mathrm{m}^{2}\right)$. The ash content (TAPPI T413 om-93 1993), bulk (TAPPI T411 om-97 1997), breaking length (ISO 1924-1 1992), Bekk smoothness (TAPPI T479 cm-99 1999), and Gurley stiffness (TAPPI T543 om-00 2000) of the handsheets were measured. 


\section{Preparation of pGCC, HCC, and pHCC}

To prepare the pre-flocculated GCC (pGCC), 0.02\% C-PAM was used as flocculant based on the dry weight GCC while stirring at 2,000 RPM. Then, $0.02 \%$ anionic polymer Perform SP7200 (MW 0.5 million g/mol. -5.0 - -3.0 meq/mol. (Solenis, USA) was added to the GCC. As soon as the GCC flocs were formed, their size was measured by using the FlowCAM $^{\circledR}$. The FlowCAM ${ }^{\circledR}$ dynamic imaging particle analyzer (Benchtop B3 Series, Fluid Imaging Technologies Inc., Scarborough, Maine, USA) measured the volume moment mean values $(\mathrm{D}[4,3])$ as average floc diameter. A few drops of the pGCC were dried on a sample holder for taking micrographs by using the scanning electron microscope (S-4800 model, Hitachi, Ltd., Tokyo, Japan). The pGCC was used to make handsheet paper.

To prepare HCC, 5.3, 16.9, 28.1, and $39.3 \mathrm{~g}$ of calcium oxide were mixed with 90 , 70,50 , and $30 \mathrm{~g}$ of GCC in water, respectively. Then, $0.03 \% \mathrm{C}-\mathrm{PAM}$ and $0.04 \%$ anionic polymer based on the dry weight of the solid were sequentially added to cause preflocculation while stirring at 2,000 rpm. After the pre-flocculation, carbon dioxide was injected into the pre-flocculated flocs at $35{ }^{\circ} \mathrm{C}$ initially, until a neutral $\mathrm{pH}$ was reached while stirring at $350 \mathrm{rpm}$. Finally, the HCCs were formed, and named as HCC10, HCC30, HCC50, and HCC70, respectively. HCC50 consisted of $50 \mathrm{~g}$ of GCC, along with $50 \mathrm{~g}$ of the newly formed PCC by the reaction. Only the HCC50 was used to make handsheet paper for comparison with the $\mathrm{pHCCs}$, and the others were used for their diameter study.

To prepare $\mathrm{pHCC}, 90,70,50$, and $30 \mathrm{~g}$ of GCC were pre-flocculated first by using $0.02 \%$ C-PAM and $0.02 \%$ anionic polymer, respectively, at 2,000 rpm and after preflocculation, calcium hydroxide of $6.4,19.2,32.1$, and $44.9 \mathrm{~g}$ were added to the respective pre-flocculated GCCs. Carbon dioxide was injected into the mixture of the pre-flocculated GCCs and the calcium hydroxide at $35^{\circ} \mathrm{C}$ until neutral $\mathrm{pH}$ was reached. For pHCC, two stirring speeds were tried such as 350 and 1,000 rpm to see whether their properties were changed by the different turbulence intensity. The final products were called as pHCC10, pHCC30, pHCC50, and pHCC70, and the newly formed PCCs to the pHCCs were 10, 30, 50 , and $70 \mathrm{wt} \%$, respectively. The sizes and shapes of the HCC, and pHCCs were determined using FlowCAM ${ }^{\circledR}$ and a scanning electron microscope, respectively. All the pHCCs prepared were used to make handsheet papers.

\section{RESULTS AND DISCUSSION}

\section{Morphology}

Micrographs of the pre-flocculated GCC and the pHCCs are presented in Fig. 1. For comparison, a micrograph of the GCC used in this study is presented (Fig. 1 (j)), where very small size filler particles were present randomly all over the micrograph. They were not attached to the larger floc by pre-flocculation or by in-situ pHCC formation process. For the pHCC10 (Fig. 1(b) and (c)), the pHCC particles looked like an aggregation of the GCC particles and very similar to the shape of the pre-flocculated GCC (a). From pHCC10 to pHCC70, the granules consisting of the pHCC surfaces were getting smaller and the GCC particles were disappeared on the surfaces. The GCC particles in pHCC70 ((h) and (i)) were located inside the pHCCs. If so, the brightness of the pHCC should be very high because of the presence of newly formed PCC on the surface. The turbulence difference at the reaction condition ( 350 and 1,000 rpm) did not cause different shapes of the pHCCs in the micrographs in Fig. 1. There showed almost no very small size particles in the 
micrographs that might disturb the formation of hydrogen bonding between wood fibers while very high portion of the GCC usually consisted of very small size free particles (Choi and Seo 2016).
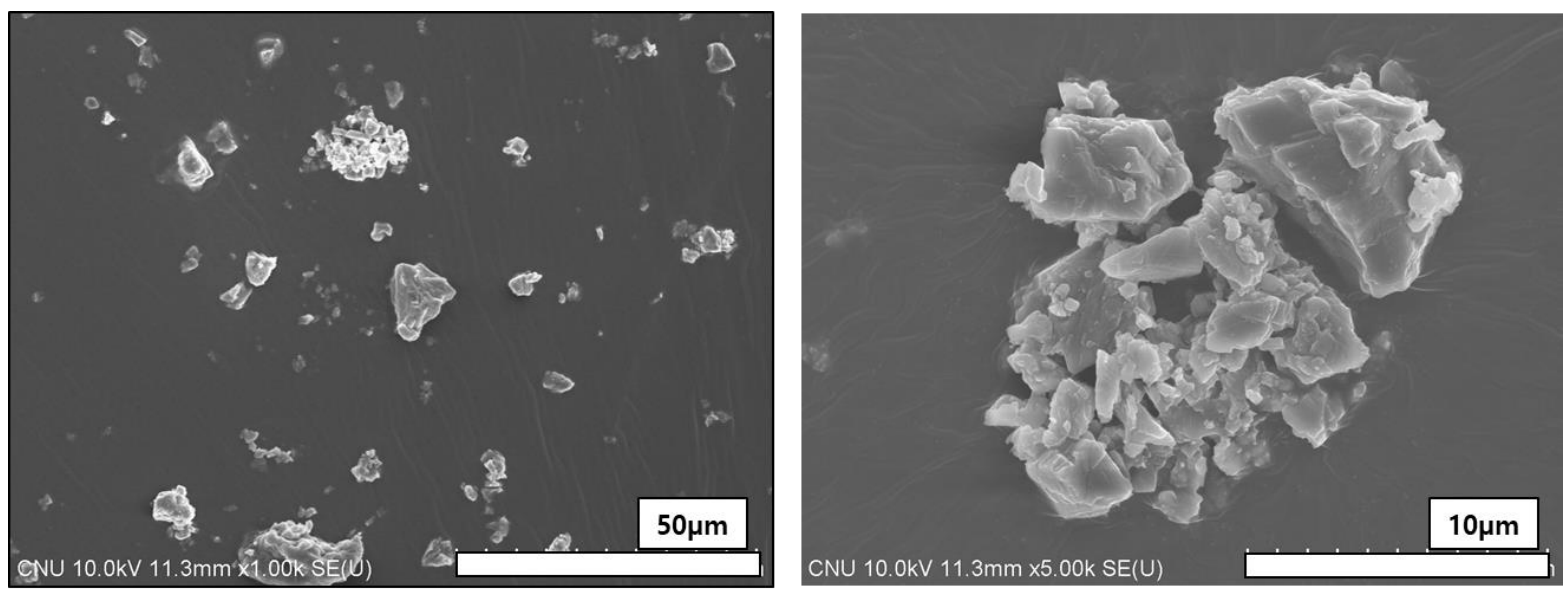

a) pGCC

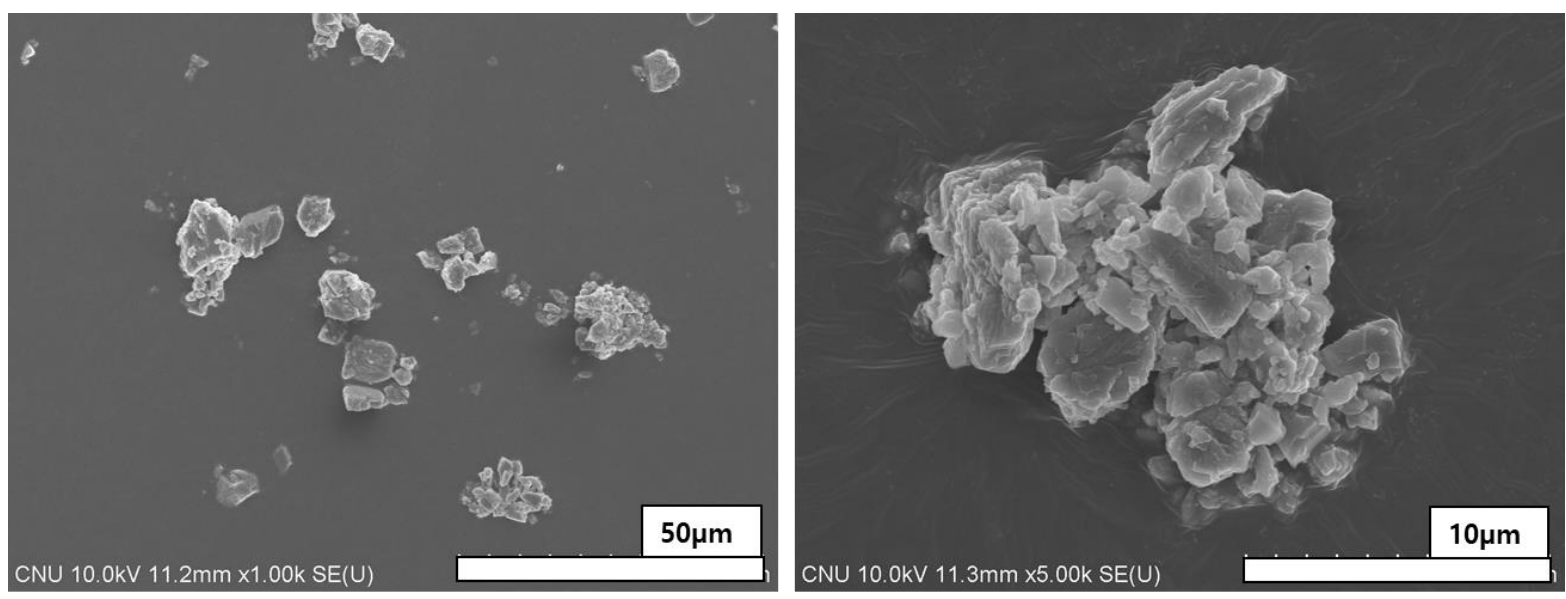

b) $\mathrm{pHCC} 10-350$
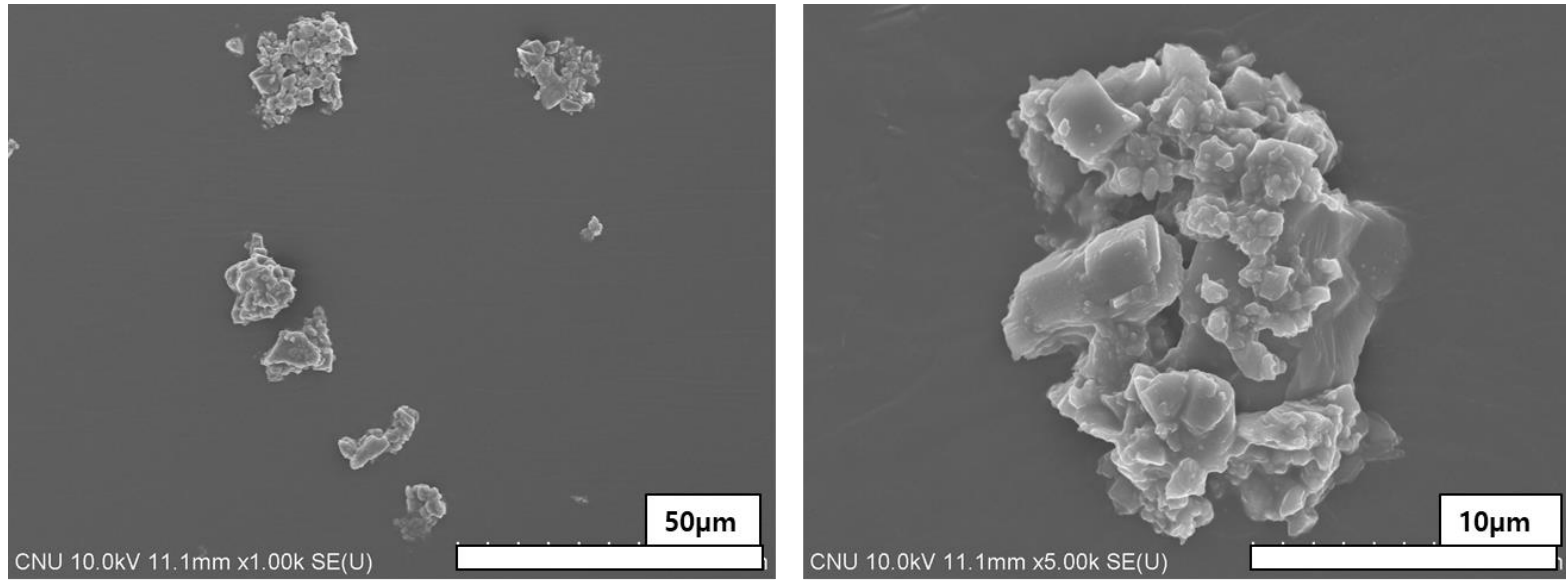

c) $\mathrm{pHCC} 10-1000$ 


\section{bioresources.com}

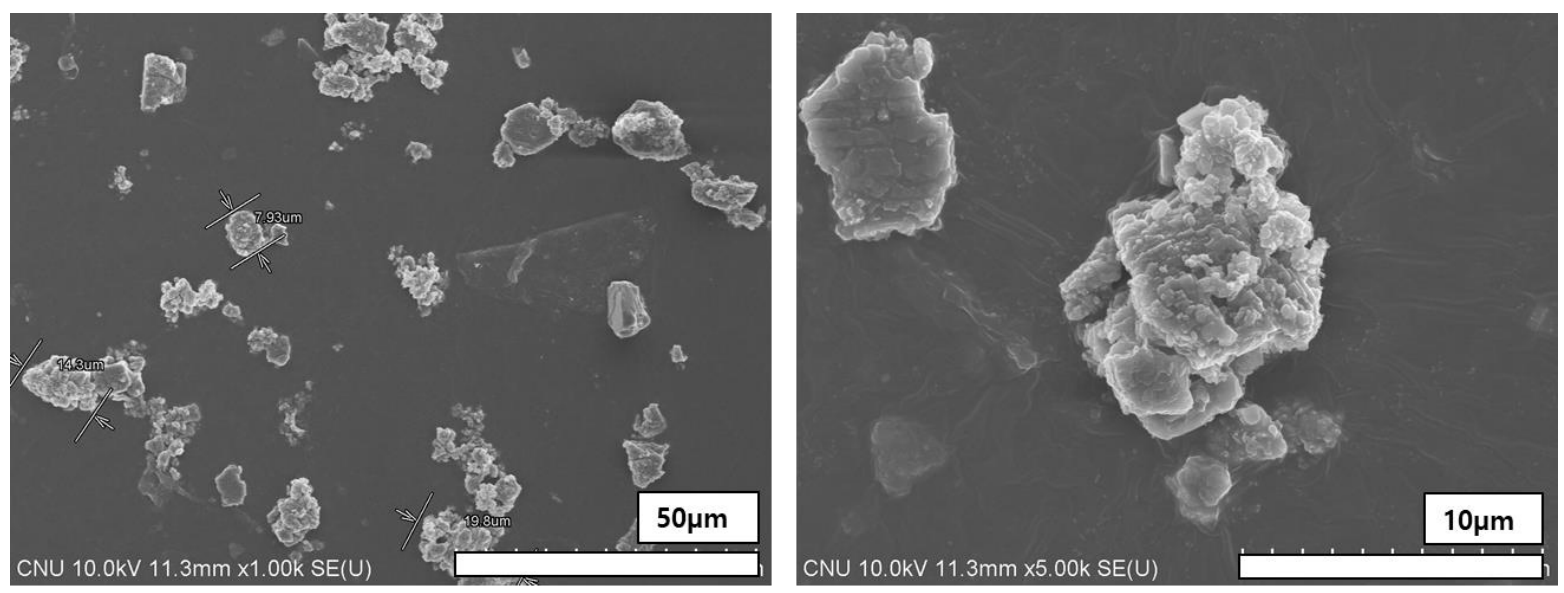

d) $\mathrm{pHCC} 30-350$
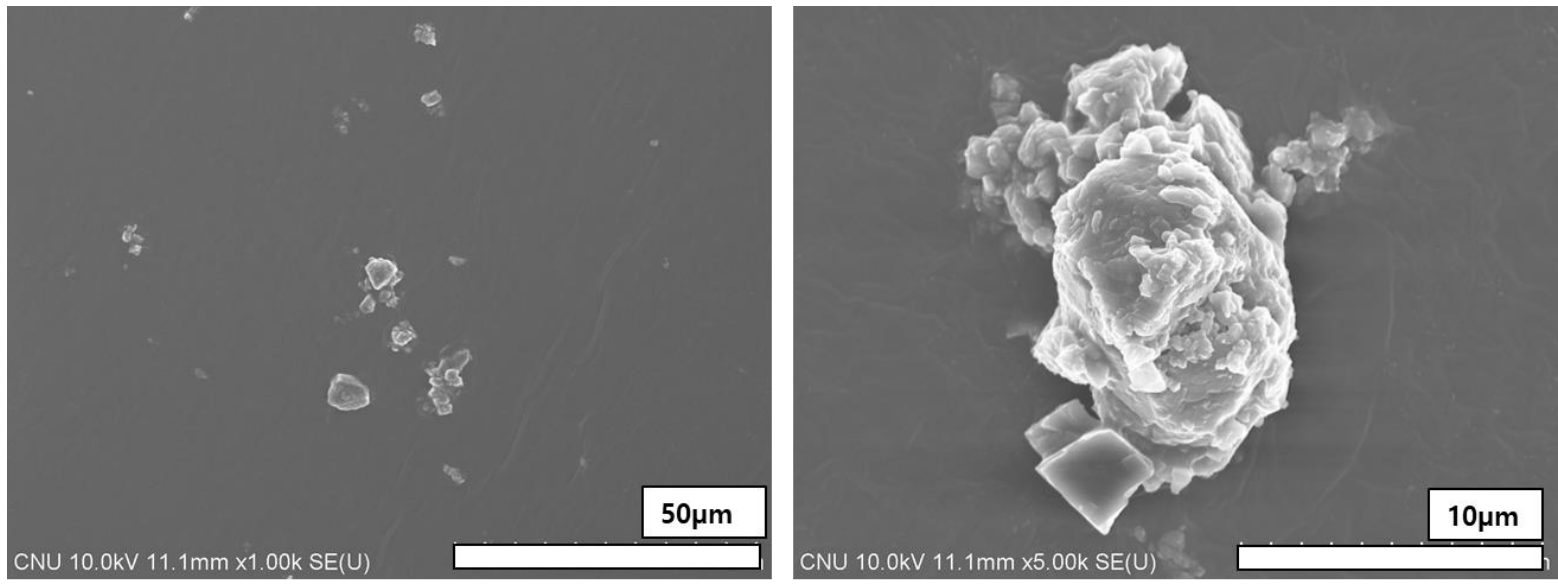

e) $\mathrm{pHCC} 30-1000$

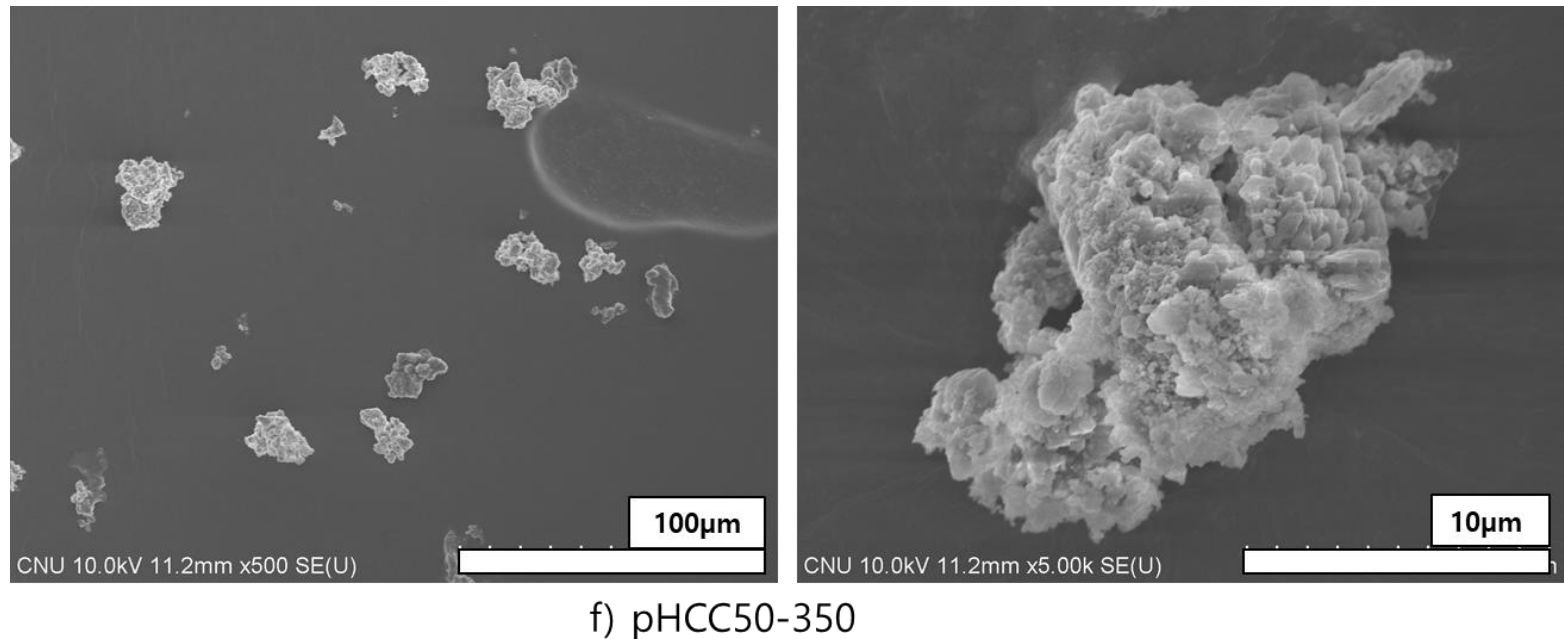




\section{bioresources.com}

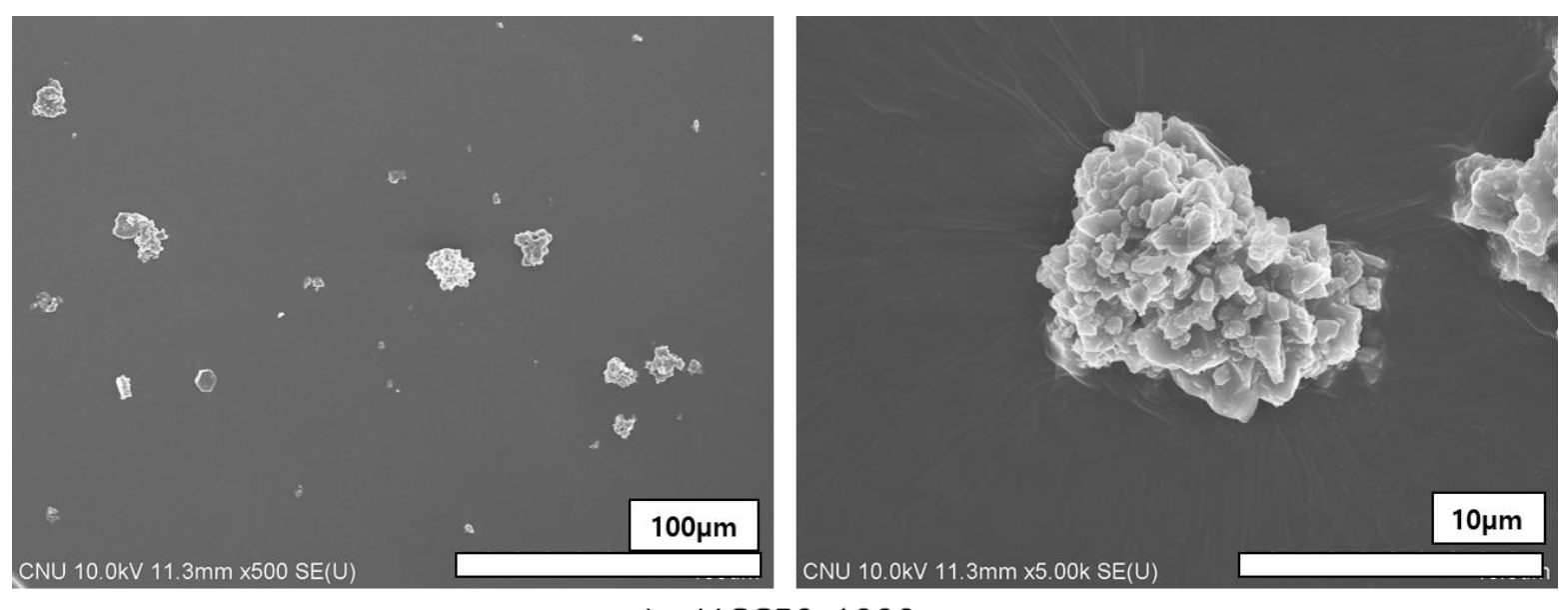

g) $\mathrm{pHCC} 50-1000$

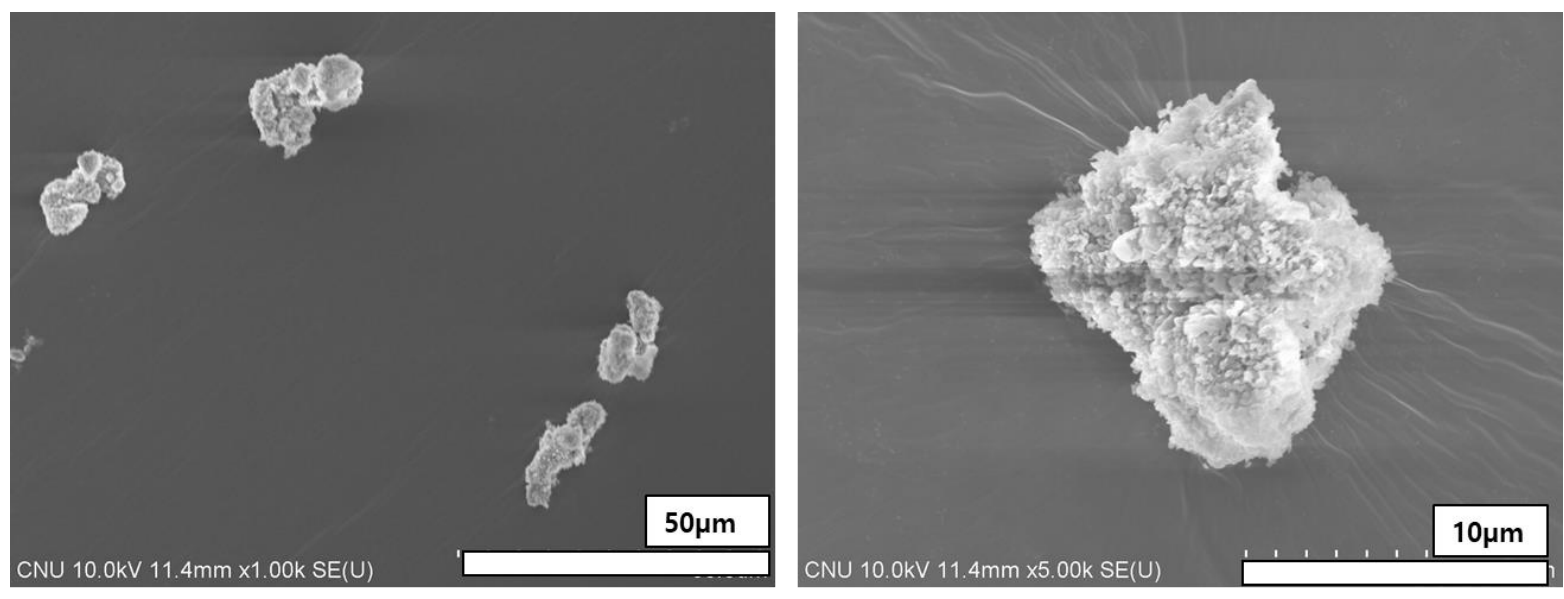

h) $\mathrm{pHCC} 70-350$
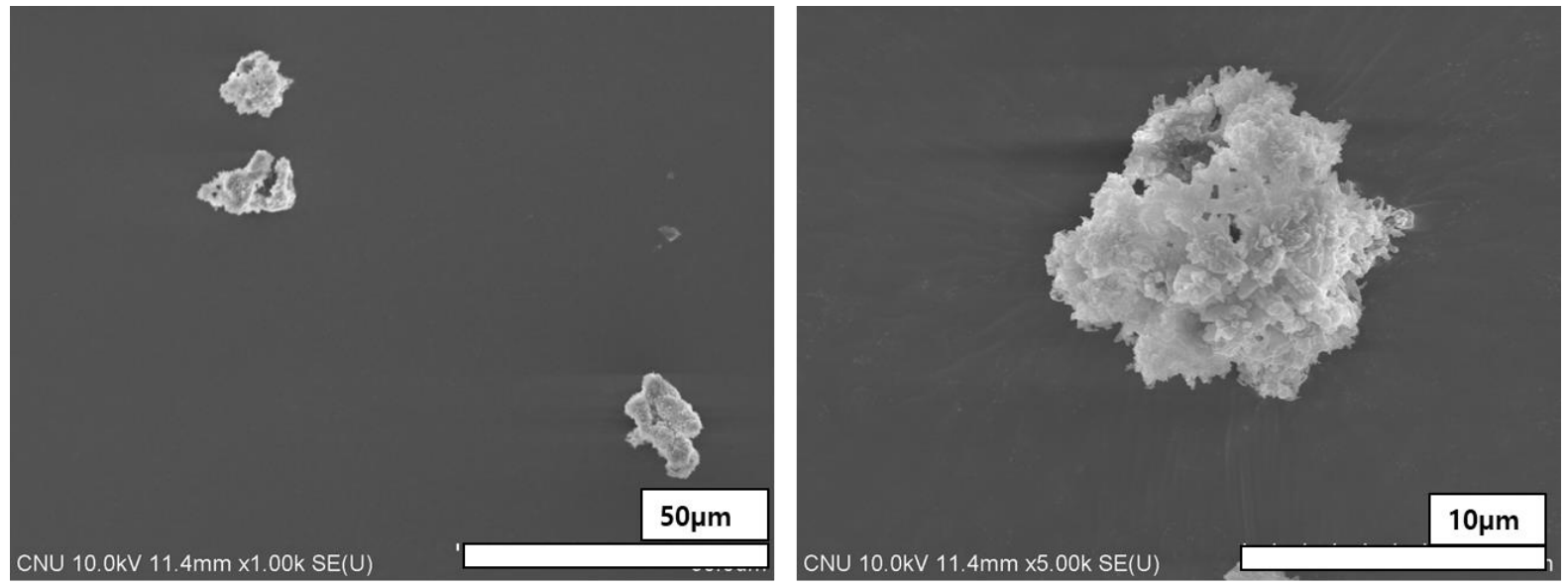

i) $\mathrm{pHCC} 70-1000$ 


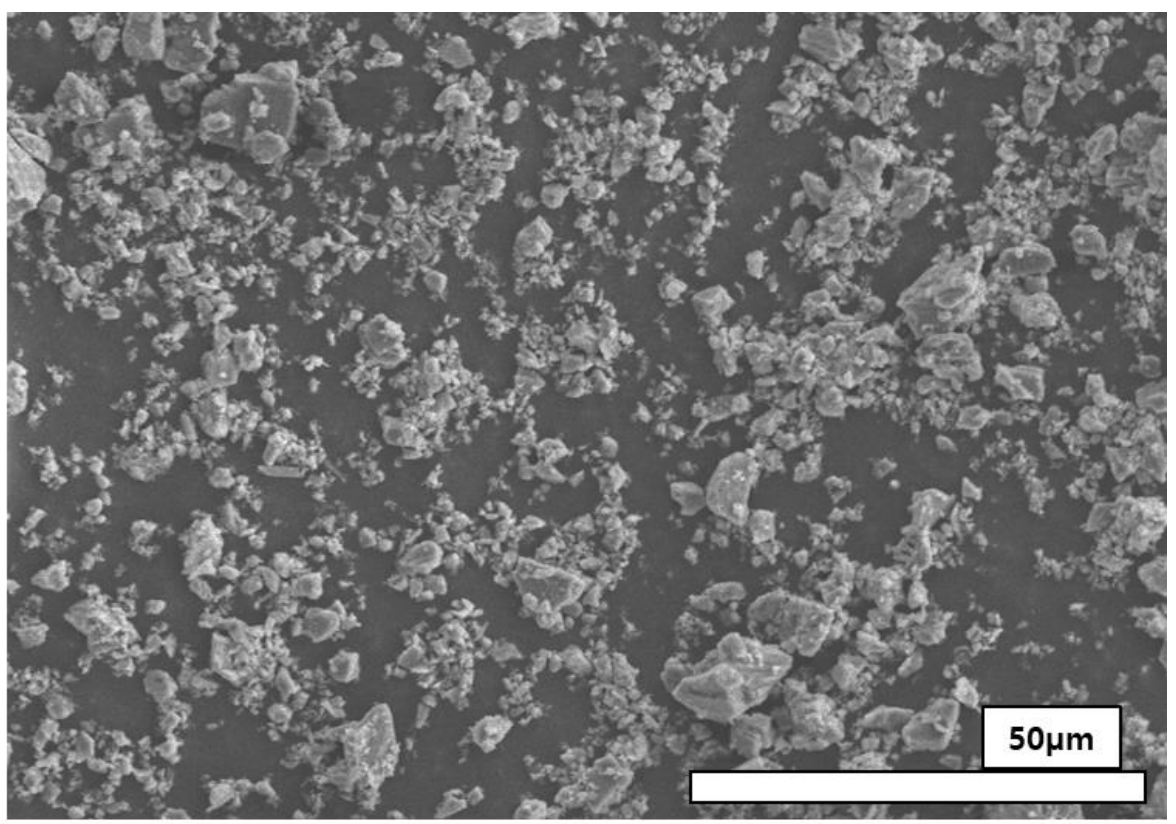

(j) GCC

Fig. 1. Micrographs of the GCC, the pre-flocculated GCC (pGCC) and the pHCCs prepared at two different turbulence. (a) pGCC: pre-flocculated GCC, (b) pHCC10-350: post HCC with $10 \%$ newly formed PCC at $350 \mathrm{rpm}$, (c) pHCC10-1000: pHCC10 formed at 1,000 rpm, (d) pHCC30-350: pHCC10-350 except 30\% newly formed PCC, (e) pHCC30-1000: pHCC10-1000 except 30\% newly formed PCC,(f) pHCC50-350: pHCC10-350 except 50\% newly formed PCC, (g) pHCC50-1000: pHCC10-1000 except 50\% newly formed PCC, (h) pHCC70-350: pHCC10-350 except 70\% newly formed PCC, (i) pHCC70-1000: pHCC10-1000 except 50\% newly formed PCC, (j) GCC.

25

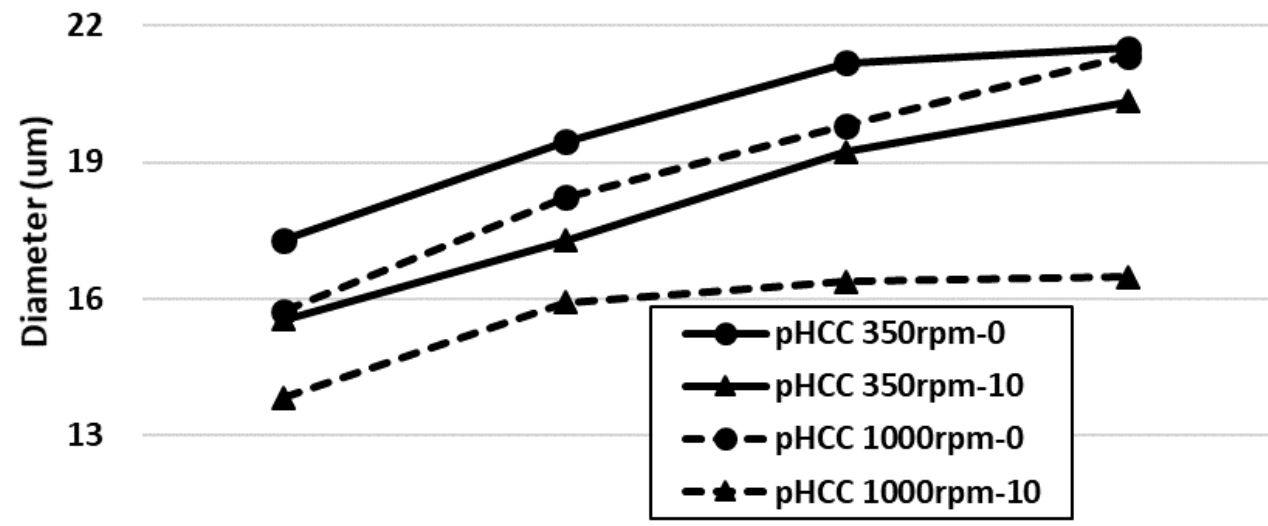

10

pHCC 10

pHCC 30

pHCC 50

pHCC 70

Sample names

Fig. 2. Diameter changes of the pHCC particles prepared at different turbulence and the effects of storing them for 0 and 10 days 
The pHCC particles prepared at different turbulence caused large a difference in the diameters as shown in Fig. 2. The diameter of the pHCC particles prepared at higher turbulence $(1,000 \mathrm{rpm})$ were smaller than those prepared at low turbulence $(350 \mathrm{rpm})$. The diameter of the pHCC increased as the portion of the newly formed PCC in pHCCs were increased. This diameter increased without the accompanying generation of small size particles and increased paper bulk without causing a decrease of bonding within the paper (Choi and Seo 2016). When the pHCCs were stored for 10 days after preparation while stirring at $100 \mathrm{rpm}$, the diameter of the pHCC particles decreased significantly. For the case of the pGCC, its diameter was initially $40.4 \mu \mathrm{m}$ at preparation stage and was changed to $12.9 \mu \mathrm{m}$ after 10 days of storage. There were many separated small size particles for the pHCCs due to collision and abrasion between $\mathrm{pHCC}$ particles during the storage, and the small size particles might cause lowering of paper bonding properties. Lack of hydrogen bonding between wood fibers usually decreases tensile strength of the paper and increases paper bulk.

The diameter changes of the HCC and pHCC are presented in Fig. 3. The diameter of HCC decreased as the portion of the newly formed PCC increased; however, for the $\mathrm{pHCCs}$, the diameter increased.

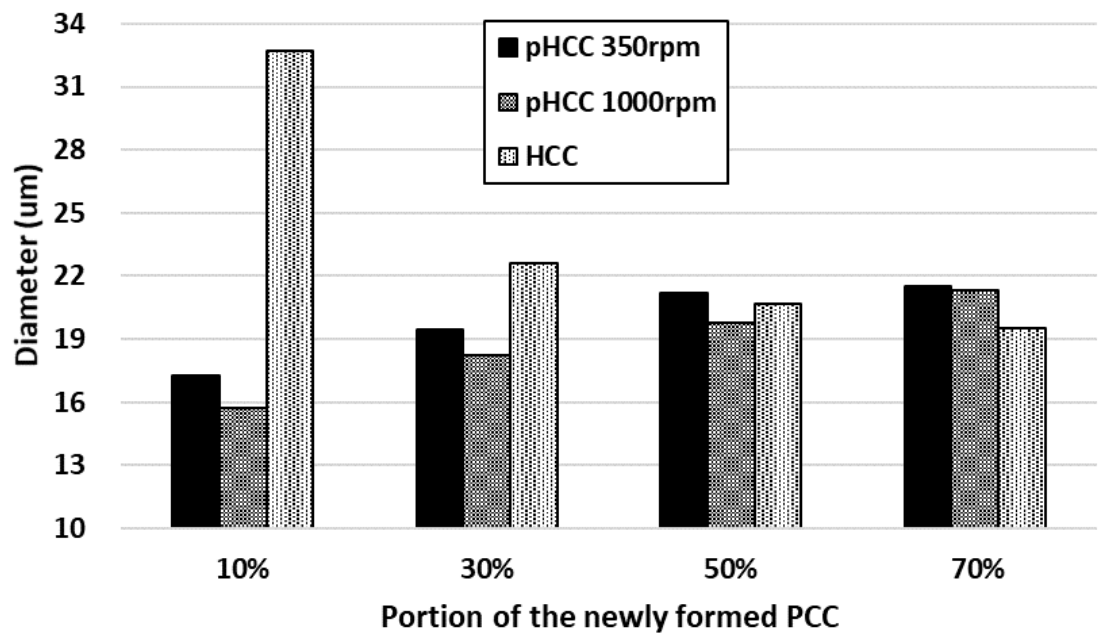

Fig. 3. Diameter of the HCCs and the pHCCs according to the increase of the newly formed PCC at preparation stage

\section{Physical and Strength Properties and Storage Stability}

Physical and strength properties of pHCCs are compared in Figs. 4 and 5. The bulk of the paper containing pHCCs in Fig. 4(a) increased as the portion of the newly formed PCC in pHCCs increased, similar to the increase of the diameters of pHCC in Figs. 2 and 3 . The pHCCs stored for 10 days with stirring caused separation of very small particles from the surface of the pHCC particles and also caused decrease the diameter of the pHCCs. The results suggested that the storage of pHCC under the stirring action generated separated small size particles that decreased the bonding between wood fibers and caused diameter decrease of pHCCs. Decreased inter-fiber bonding in paper also caused increase of bulk in the paper. The pGCC gave the same pattern of bulk increase (Fig. 4(a)). It was also found that the bulk of the papers containing pHCCs began to increase greatly along with the diameter increase of $\mathrm{pHCC}$ when the portion of the newly formed PCC were over $50 \%$. 
In Fig. 4(b), the breaking lengths of all papers containing pHCC were much higher than that of the GCC containing paper $(\boldsymbol{\Delta})$. Another observation was that the paper which contained the pHCCs formed at 1,000 rpm and were stored for 10 days gave large drops of breaking length, while the pHCCs formed at $350 \mathrm{rpm}$ showed less differences by the storage time. The pHCC storage while stirring caused the generation of small size particles and decreased the diameter of the pHCCs, which then resulted in a decrease of breaking length. The pre-flocculated GCC ( $\square)$ gave a larger drop of breaking length after 10 days of storage ( $\square$ ) than that of the pHCCs due to its nature of polymer-aided, soft aggregation.

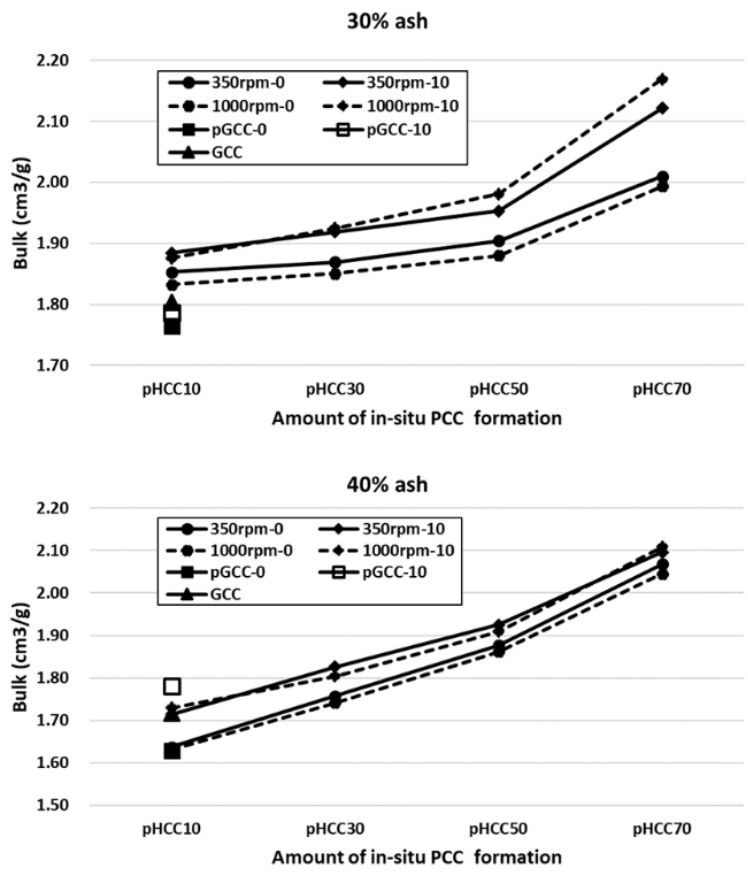

(a) Bulk $\left(\mathrm{cm}^{3} / \mathrm{g}\right)$

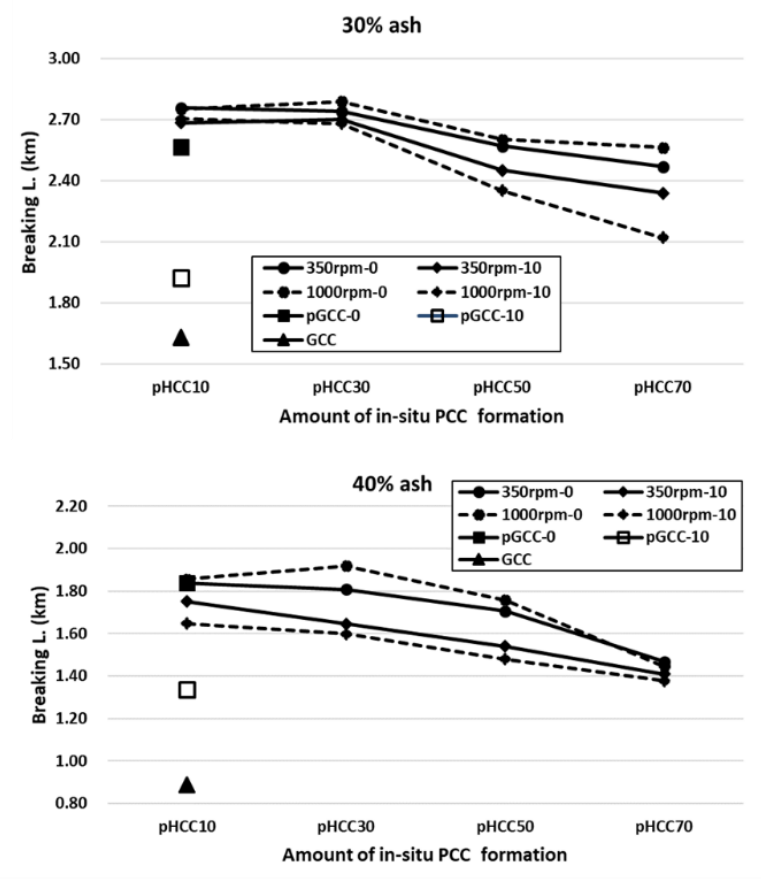

(b) Breaking length $(\mathrm{km})$

Fig. 4. Bulk and breaking length properties of the papers containing pHCCs with four different levels of the newly formed PCCs including the properties after 0 and10 days of pHCC storage

The stiffness changes of the papers containing pHCCs (Fig. 5 (a)) followed the patterns of the bulk changes in Fig 4 (a) except more straight line in stiffness cases. It looked reasonable because the stiffness was affected by the cube of the thickness of the paper. As in the case of bulk, the stiffness values of the papers containing the pHCCs stored for 10 days were higher than for those containing fresh pHCCs and the stiffness increased as the portion of the newly formed PCC in pHCCs increased.

The Bekk smoothness of the papers containing pre-flocculated GCC and pHCCs were not much different even though the diameter of the GCC (aver. dia. $2 \mu \mathrm{m}$ ) was much smaller than those of the pHCCs, as shown in Fig. 5 (b). The pHCCs are deformable and conformable to the compressive pressure applied during the papermaking process just like HCC cases (Choi and Seo 2016). Furthermore, the Bekk smoothness of the papers containing pre-flocculated GCC and pHCCs were all lowered after 10 days of storage. This consistent behavior was not expected but needs further study to explain. This lab study was done without calendaring and could not fully represent the smoothness behavior of the pHCCs. However, there was no significant differences in paper smoothness between GCC and the pHCCs despite more than 10 times diameter differences. 

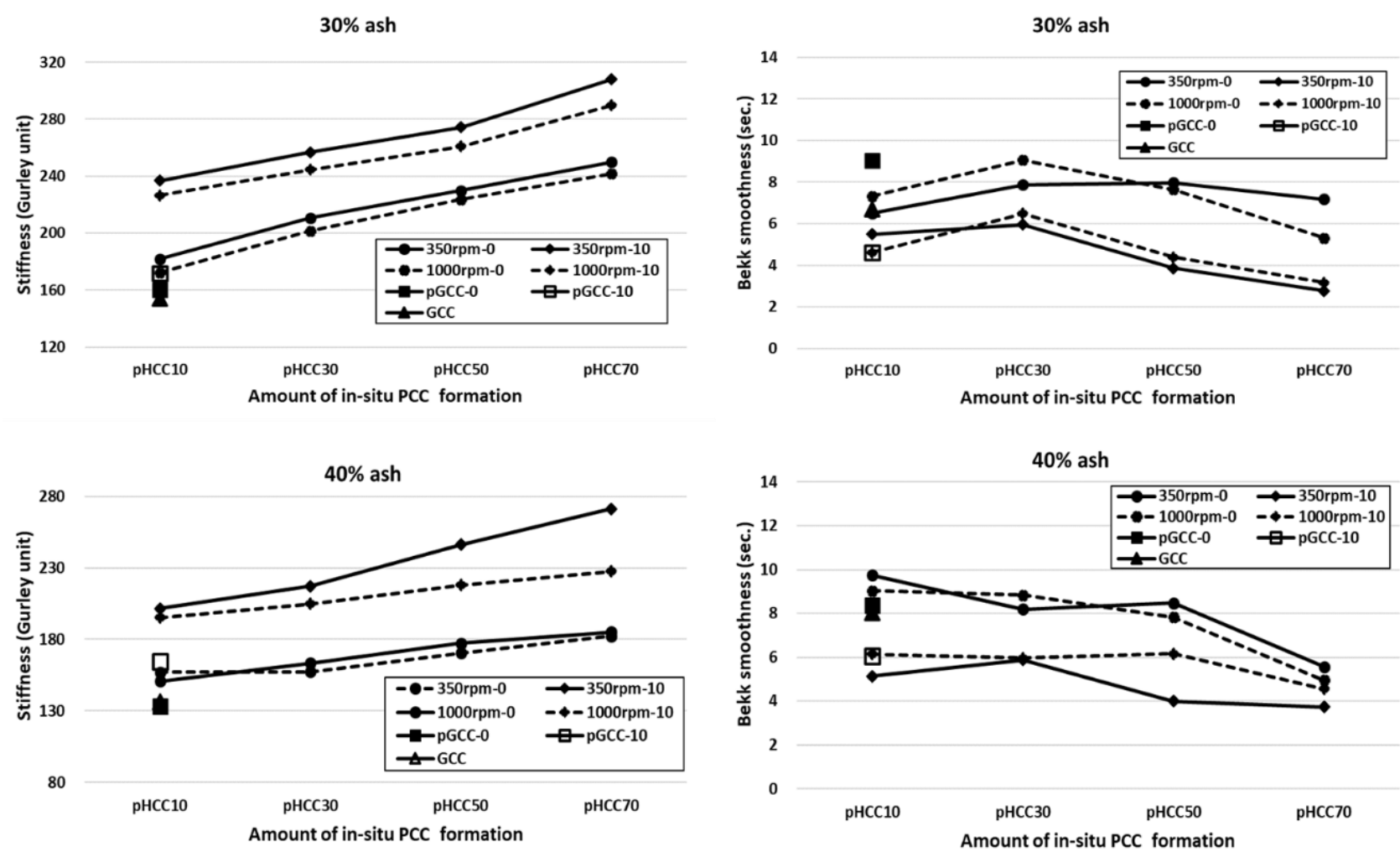

(a) Stiffness (Gurley unit)

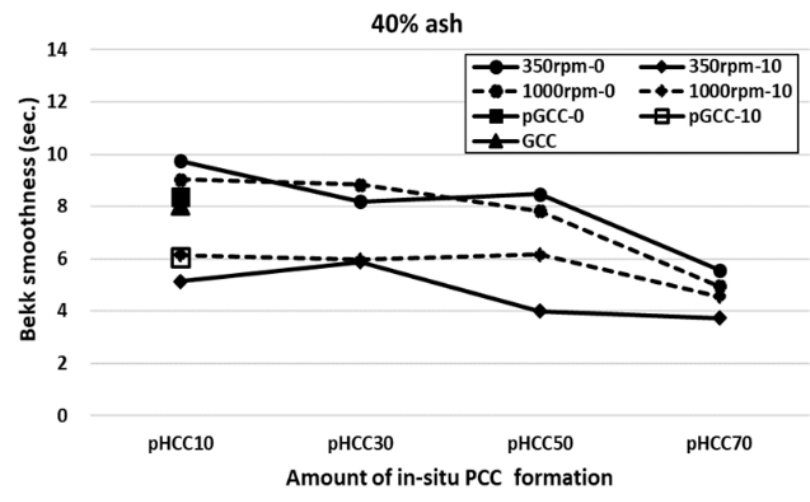

(b) Bekk smoothness (s)

Fig. 5. Stiffness and Bekk smoothness properties of the papers containing pHCCs with four different levels of the newly formed PCCs including the properties after 0 and10 days of storage

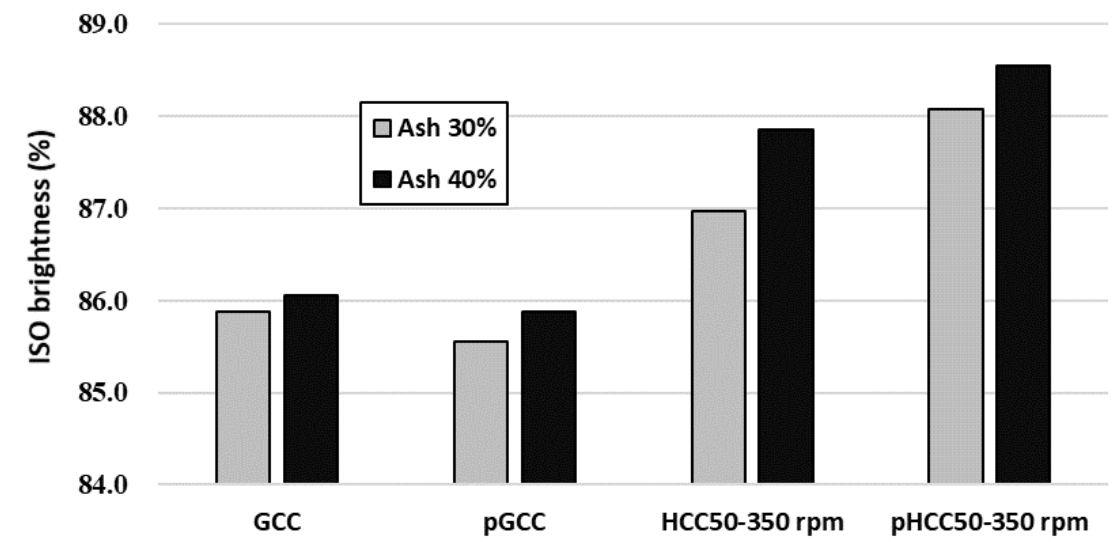

Fig. 6. ISO brightness of the papers containing different fillers 
The brightness values of the papers are shown in Fig. 6, where the pHCC50-350 $\mathrm{rpm}$, which consisted of $50 \mathrm{wt} \%$ of the newly formed PCC and stirred at $350 \mathrm{rpm}$ during reaction, gave the highest value, and the pGCC gave the lowest. The newly formed PCCs were located on the surface of the pHCC. HCC50-350 rpm also consisted of $50 \mathrm{wt} \%$ of the newly formed PCC, but in this case, the newly formed PCC and GCC were mixed together. The pre-flocculated GCCs (pGCCs) consisted of an aggregation of GCCs connected by ionic polymers and seemed to lose some scattering surface area per mass when compared to the individually separated GCCs. Less scattering surface area caused less scattering of visible light and also caused slightly lower opacity and brightness (Fig. 6) than the GCC.

\section{Savings in Wood Fibers}

If there occurred a superior property in paper manufacture, it needs to be properly compensated in such a way as savings of raw materials, production cost, or energy. The possibility of using pHCCs for wood fiber replacement by $10 \%$ is shown in Fig. 7 when compared to the case of using GCC that were commercially used in the paper mills. Three key properties were presented, and the paper containing $40 \%$ of the samples named as pHCC50-350 and pHCC50-1000 was better in all three properties than the paper containing $30 \%$ of GCC. Pre-flocculation method was incapable of replacing $10 \%$ wood fibers due to low bulk and low stiffness. The cost of producing HCC and pHCC should be in the midpoint of GCC and PCC.

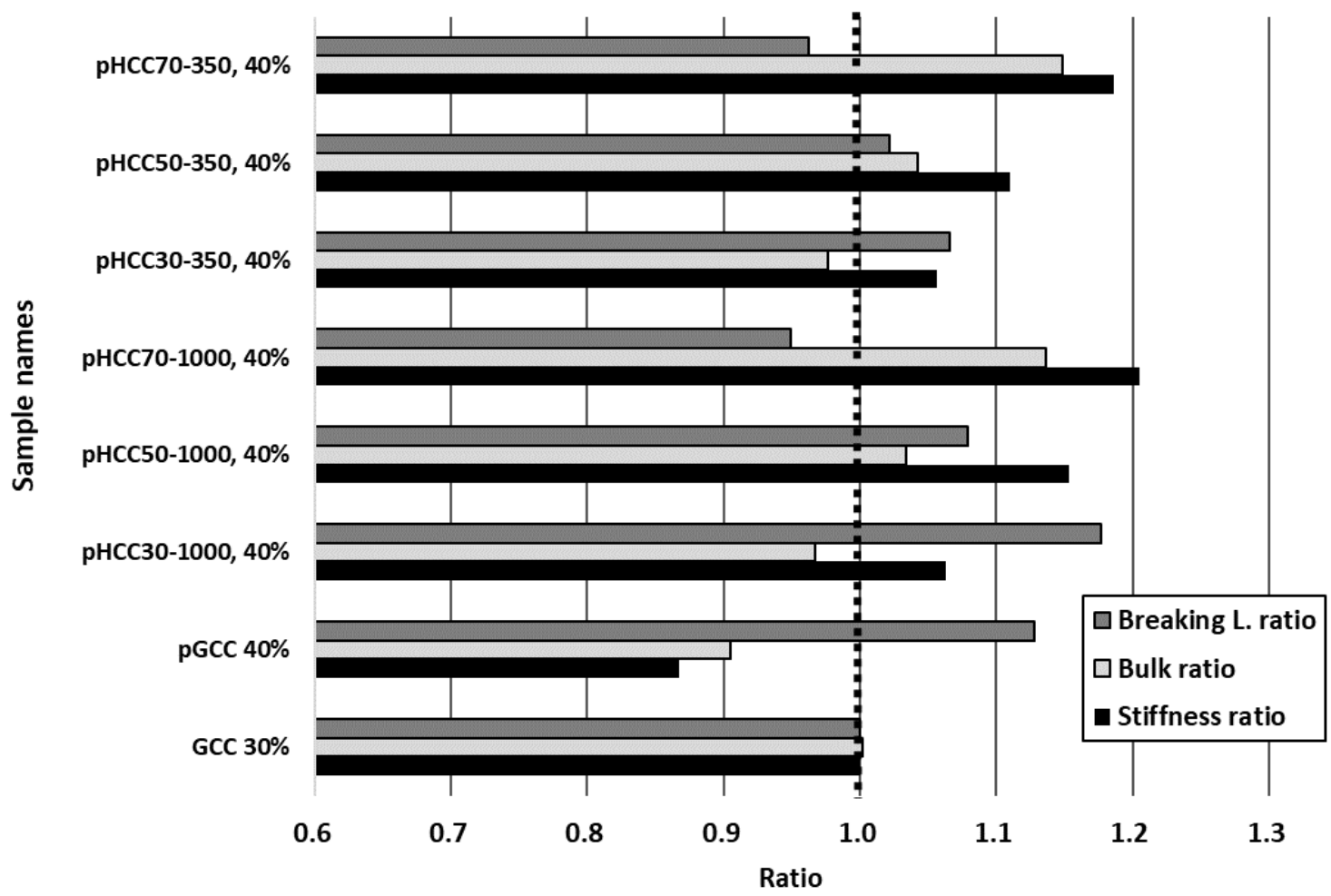

Fig. 7. Comparison of physical property ratios of the papers containing $40 \% \mathrm{pHCCs}$ against those of the paper containing $30 \%$ GCC. 


\section{CONCLUSIONS}

1. The post hybrid calcium carbonate ( $\mathrm{pHCC}$ ) gave much superior properties in breaking length, bulk, and stiffness than the ground calcium carbonate (GCC) and it was possible to add $10 \%$ more filler to the paper without lowering those properties and without lowering smoothness when compared to the GCC containing paper.

2. The increase of the portion of the newly formed precipitated calcium carbonate (PCC) in pHCCs increased the diameter of pHCCs and left almost no small size particles free in suspension. Large diameter of the pHCC caused bulk increase of the paper and no presence of the absence of small size particles led to higher paper bonding properties.

3. Higher turbulence during $\mathrm{pHCC}$ preparation stage decreased the diameter and the diameter stability of the pHCC for storage.

4. Storing $\mathrm{pHCC}$ while stirring for 10 days caused generation of small particles from the pHCC surfaces and decrease of pHCC diameter. The small particles decreased paper bonding properties and increased paper bulk.

5. Saving wood fibers by replacing with inexpensive inorganics such as pHCC may result in production cost and drying energy savings.

\section{ACKNOWLEDGMENTS}

This research was supported by the National Strategic Project-Carbon Upcycling of the National Research Foundation of Korea (NRF) funded by the Ministry of Science and ICT (MSIT), the Ministry of Environment (ME), and the Ministry of Trade, Industry and Energy (MOTIE) (NRF-2019M3D8A2113265).

\section{REFERENCES CITED}

Burgess, M. S., Phipps, J. S., and Xiao, H. (2000). "Flocculation of PCC induced by polymer/microparticle systems: Floc characteristics," Nord. Pulp Paper Res. J. 15(5), 572-578. DOI: 10.3183/npprj-2000-15-05-p572-578

Chauhan, V. S., and Bhardwaj, N. K. (2014). "Cationic starch preflocculated filler for improvement in filler bondability and composite tensile index of paper," Ind. Eng. Chem. Res. 53(29), 11622-11628. DOI: 10.1021/ie502008d

Choi, J. S., and Seo, Y. B. (2016). "Development of hybrid calcium carbonate for high loading paper (III), post hybrid calcium carbonate," J. Korea TAPPI 48(6), 55-61.

Choi, J. S., Kang, D. S., Han, J. S., and Seo, Y. B. (2018). "Property development mechanism of hybrid calcium carbonate," ACS Sustainable Chem. Eng. 7(1), 15381544. DOI: 10.1021/acssuschemeng.8b05304

Fuente, E., Blanco, A., Negro, C., Pelach, M. A., Mutje, P., and Tijero, J. (2005). "Study of filler flocculation mechanisms and floc properties induced by polyethylenimine," Ind. Eng. Chem. Res. 44(15), 5616-5621. DOI: 10.1021/ie0503491

Gaudreault, R., Cesare, N. D., Weitz, D., and van de Ven, T. G. M. (2009). "Flocculation kinetics of precipitated calcium carbonate," Colloids Surf. A Physicochem. Eng. Asp. 340(1-3), 56-65. DOI: 10.1016/j.colsurfa.2009.03.008 
Gray, S. R., and Ritchie, C. B. (2006). "Effect of organic polyelectrolyte characteristics on floc strength," Colloids Surf. A Physicochem. Eng. Asp. 273(1-3), 184-188. DOI: 10.1016/j.colsurfa.2005.08.020

ISO 1924-1(1992). "Paper and board — Determination of tensile properties — Part 1: Constant rate of loading method," International Organization for Standardization, Geneva, Switzerland.

Jung, J. K., and Seo, Y. B. (2015). "Development of hybrid calcium carbonate for high loading paper (I), manufacture and application of hybrid calcium carbonate," J. Korea TAPPI 47(4), 30-37.

Lee, K. H., and Lee, H. L. (2006). "Preflocculation of GCC with cationic PAM and cationic starch and the influence of their dosage and shear rate on prefloc size," $J$. Korea Tappi 38(4), 1-9.

Modgi, S., McQuaid, M. E., and Englezos, P. (2006). "Interaction of precipitated calcium carbonate with starch in distilled and deionized water and process water," Nord. Pulp Paper Res. J. 21(5), 716-723. DOI: 10.3183/npprj-2006-21-05-p716-723

Seo, D., Im, W. H., Youn, H. J., and Lee, H. L. (2012). "The effects of process variables for GCC pre-flocculation on floc and handsheet properties," Nord. Pulp Paper Res. J. 27(2), 382-387. DOI: 10.3183/npprj-2012-27-02-p382-387

TAPPI T411 om-97 (1997). "Thickness (caliper) of paper, paperboard, and combined board," TAPPI press, Atlanta, GA.

TAPPI T413 om-93 (1993). “Ash in wood, pulp, paper and paperboard," TAPPI Press, Atlanta, GA.

TAPPI T479 cm-99 (1999). "Smoothness of paper (Bekk method)," TAPPI Press, Atlanta, GA.

TAPPI T543 om-00 (2000). "Bending resistance of paper (Gurley-type tester)," TAPPI Press, Atlanta, GA.

Yeung, A., Gibbs, A., and Pelton, R. (1997). "Effect of shear on the strength of polymerinduced flocs," J. Colloid Interface Sci. 196(1), 113-115. DOI:

$10.1006 /$ jcis. 1997.5140

Article submitted: July 7, 2021; Peer review completed: September 28, 2021; Revised version received and accepted: September 29, 2021; Published: October 1, 2021.

DOI: 10.15376/biores.16.4.7716-7728 